\title{
Ergonomia na central de material e esterilização
}

\author{
Ergonomics at the central supply \\ Ergonomía en la central de sterilización
}

Recebido: 30/07/2021 | Revisado: 02/08/2021 | Aceito: 06/08/2021 | Publicado: 11/08/2021

Isabella Barbosa da Silva

ORCID: https://orcid.org/0000-0003-2477-4572 Universidade Veiga de Almeida, Brasil

E-mail: bebellabar@gmail.com

Jéssica Sanxes de Paiva

ORCID: https://orcid.org/0000-0001-6798-1681 Universidade Veiga de Almeida, Brasil

E-mail: jessicasanxes@gmail.com

Maria Virginia Godoy da Silva

ORCID: https://orcid.org/0000-0003-3980-042X

Universidade Veiga de Almeida, Brasi

E-mail: godoydasilva@ terra.com.br

Leandro Andrade da Silva

ORCID: https://orcid.org/0000-0003-3213-5527

Universidade Veiga de Almeida

E-mail: proflandrade@gmail.com

Carolina Pimentel Machado

ORCID: https://orcid.org/0000-0001-6883-8967 Universidade Veiga de Almeida carol.machado@outlook.com.br

Gláucya Lima Daú

ORCID: https://orcid.org/0000-0002-1948-9868 Universidade Federal do Estado do Rio de Janeiro, Brasil E-mail: glaucyadau @gmail.com

Karen Corrêa do Canto

ORCID: https://orcid.org/0000-0001-5580-3191 Universidade Veiga de Almeida, Brasil

E-mail: kkarencorrea@gmail.com

Gyovana Regis de Oliveira

ORCID: https://orcid.org/0000-0002-4809-1736 Universidade Veiga de Almeida, Brasil

E-mail: gyovanaregis@gmail.com

Luiza Campos de Oliveira Batista

ORCID: https://orcid.org/0000-0003-1566-1330

Universidade Veiga de Almeida, Brasi luizacamposrj@gmail.com

Mielli Reis Araújo de Sousa

ORCID: https://orcid.org/0000-0002-4600-5101

Universidade Veiga de Almeida, Brasil

E-mail: miellisousa97@hotmail.com

\section{Resumo}

A Central de Material e Esterilização (CME), destaca-se como setor complexo do hospital para os profissionais exercerem suas funções, apresentando riscos químicos, físicos, mecânicos, biológicos e ergonômicos. Os riscos ergonômicos estão associados à organização do trabalho, mobiliário, equipamentos, levantamento e transporte manual de peso, jornada dupla de trabalho, relações conflituosas, monotonia ou ritmos intensos no serviço. Objetivo: analisar as produções científicas sobre riscos ergonômicos na CME. Metodologia: revisão integrativa da literatura de acordo com a estratégia (PICo) e procedimentos metodológicos definidos pelo Preferred Reporting Items for Systematic Reviews and Meta-Analyses (PRISMA). Busca bibliográfica realizada no período de abril a maio de 2021 , sem definição de tempo, nos idiomas português, inglês e espanhol. Foram selecionados 9 estudos para análise Bases de dados: Sistema Online de Busca e Análise de Literatura Médica (MEDLINE)/PubMed, Literatura Latino-Americana e do Caribe em Ciências da Saúde (Lilacs) e Base de Dados em Enfermagem (BDEnf) da Biblioteca Virtual em Saúde (BVS). Resultados: A CME oferece muitos riscos para seus trabalhadores: ambientes de trabalho inadequados, falta de insumos e equipamentos; esforço físico, postura inadequada, levantamento e transporte manual de peso, trabalho diuturno, repetitividade. Outros riscos pontuados: jornada de trabalho dupla, turnos de trabalho exaustivos, movimentos repetitivos, esforço físico, postura errada, mobiliário inadequado. Conclusão: A prática ergonômica, na CME minimizaria riscos mediante mobiliário 
adequado, pausa para descanso, melhor distribuição do pessoal, gestores com conhecimento de ergonomia e educação continuada preparando os funcionários, orientando-os para o tema da ergonomia, direitos e responsabilidades.

Palavras-chave: Enfermagem; Ergonomia; Esterilização; Saúde do Trabalhador.

\begin{abstract}
The Material and Sterilization Center (CME) stands out as a complex sector of the hospital for professionals to perform their functions, in which the scope presents various risks such as: chemical, physical, mechanical, biological and ergonomic. Ergonomic risks are associated with work organization, furniture, equipment, lifting, manual lifting and transport of weight, douple work shift, conflicting relationships, monotony or intense work rhythms. Objective: to analyze the scientific productions on ergonomic risks in the CME. Methodology: integrative literature review, systematized by the acronym Participant, Interest and Context (PICo) according to the strategy (PICo) and methodological procedures defined by the Preferred Reporting Items for Systematic Reviews and Meta-Analyzes (PRISMA). The bibliographic search will be carried out between April to May 2021, with no time definition, in Portuguese, English and Spanish. Nine studies were selected in the databases Online System for Searching and Analyzing Medical Literature (MEDLINE) / PubMed, Latin American and Caribbean Literature in Health Sciences (Lilacs) and Base Nursing Data (BDEnf) from the Virtual Health Library (VHL). Results: CME presents many risks for their professionals: inadequate work environment, lack of resources and equipments, physical effort, inadequate posture, lifting and carrying of heavy weights, long hours of work, repetitiveness. Other risks shown: double work journey, exhausting work shifts, repetitive movements, physical effort, wrong posture, inadequate furniture. Conclusion: Ergonomic practice at the CME would minimize these risks through adequate furniture, rest breaks, better staff distribution, managers with knowledge of ergonomics and continuing education preparing employees, guiding them to the topic ofergonomics, rights and responsibilities.
\end{abstract}

Keywords: Nursing; Ergonomics; Sterilization; Occupational Health.

\title{
Resumen
}

El Centro de Material y Esterilización (CME) se destaca como un sector complejo del hospital para que los profesionales realicen sus funciones, en el que el ámbito presenta diversos riesgos tales como: químico, físico, mecánico, biológico y ergonómico. Los riesgos ergonómicos están asociados a la organización del trabajo, mobiliario, equipamiento, elevación manual y transporte de peso, doble turno de trabajo, relaciones conflictivas, monotonía o ritmos de trabajo intensos. Objetivo: analizar las producciones científicas sobre riesgos ergonómicos en las CME, identificar las estrategias que reducenestos riesgos y revelar las causas de remoción de trabajadores de las CME asociadas a los riesgos ergonómicos. Metodología: revisión integradora de la literatura, sistematizada por las siglas Participante, Interés y Contexto (PICo) de acuerdo con la estrategia (PICo) y procedimientos metodológicos definidos por los Ítems Preferidos de Reporte para Revisiones Sistemáticasy Metanálisis (PRISMA). La búsque da bibliográfica se realizará entre abril a mayo de 2021, sin definición de tiempo, en portugués, inglés y español. En las bases de datos Sistema Online de Búsqueda y Análisis de Literatura Médica (MEDLINE) / PubMed, Literatura Latino Americana y Caribeña en Ciencias de la Salud (Lilacs) y Base Nursing Data (BDEnf) del Biblioteca Virtual en Salud (BVS). Resultado: El CME presenta muchos riesgos a sus trabajadores: ambientes de trabajo inadecuados, la falta de insumos y equipamientos, el esfuerzo físico, la postura inadecuada, el levantamiento y transporte manual del peso, el trabajo diurno y la repetitividad. Otros riesgos señalados son: la doble jornada laboral, los turnos de trabajo exhaustivos, los movimientos repetitivos, el esfuerzo físico, la postura incorrecta y el mobiliario inadecuado. Conclusión: La práctica ergonómica en el CME minimizaría estos riegos a través de mobiliario adecuado, descansos, mejor distribución del personal, gerentes con conocimiento de ergonomía y educación continua preparando a los empleados, orientándo los hacia el tema de ergonomía, derechos y responsabilidades.

Palabra clave: Enfermería; Ergonomía; Esterilización; Salud ocupacional.

\section{Introdução}

A Central de Material e Esterilização (CME) é definida como um setor responsável do hospital pelo cuidado assistencial e apoio técnico indiretos aos pacientes internados (SOBECC, 2017). A Resolução da Diretoria Colegiada n. 15 (RDC 15), de 15 de março de 2012, da Agência Nacional de Vigilância Sanitária (ANVISA), regulamenta a CME e estabelece boas práticas para o processamento de produtos para saúde (PPS) como a pré-limpeza, recepção, limpeza, secagem, avaliação da integridade e da funcionalidade; preparo, desinfecção ou esterilização; armazenamento e distribuição para as unidades consumidoras, buscando a segurança do paciente e dos profissionais dos serviços que realizam o processamento de PPS (Brasil, 2012).

A CME retrata um setor complexo para os profissionais exercerem suas funções, pois os expõem a riscos químicos, físicos, mecânicos, biológicos e ergonômicos. As ameaças à saúde ocorrem visto que o indivíduo possui interação direta com uma diversidade de matérias e substâncias em ambientes/espaços físicos impróprios, com um ritmo acelerado de 
trabalho, imposições da chefia, longo período no serviço e laboração, ou rejeição ao uso dos Equipamentos de Proteção Individual (EPI) e carência de medidas de proteção coletiva (Lima et al., 2018).

A operação e o trabalho da CME e o modo como os trabalhadores utilizam de estratégias propostas, influenciam diretamente na Saúde do Trabalhador (ST) podendo impactar no processo saúde x doença (Espindola \& Fontana, 2012). Deste modo, a exposição dos profissionais às ameaças diversas, cria a necessidade de um ambiente adequado para diminuir os riscos ocupacionais atendendo as normas oficiais de padronização, onde o indivíduo sadio deve ser o foco de ações em saúde para melhor desempenho laboral (Sousa, 2011).

Aquino et al. (2014) consideram a CME um dos ambientes no cenário hospitalar que tornam as equipes de Enfermagem mais vulneráveis aos riscos ocupacionais.

De acordo com a Associação Internacional de Ergonomia (AIE), a Ergonomia é uma disciplina relacionada ao entendimento das interações entre os seres humanos e outros elementos ou sistemas. Ela também trata da aplicação de teorias, princípios, dados e métodos a projetos a fim de otimizar o bem-estar humano e o desempenho global do sistema (IEA, 2020).

No Brasil, a Ergonomia é regida pela Norma Regulamentadora (NR) n ${ }^{\circ} 17$ da Portaria 3.214/78, do Ministério do Trabalho e Emprego (MTE) que visa estabelecer parâmetros que permitam a adaptação das condições de trabalho às características psicofisiológicas dos trabalhadores, de modo a proporcionar um máximo de conforto, segurança e desempenho eficiente (Brasil, 1978).

Para Gouveia (2016), a Ergonomia tem a finalidade de promover o desenvolvimento das circunstâncias de trabalho, tanto no sentido material, quanto nas ferramentas organizacionais e sociais, para que se possa alcançar a saúde. Os riscos ergonômicos envolvem questões associadas à organização do trabalho, mobiliário, equipamentos. Se relaciona também ao levantamento e transporte manual de peso, jornada dupla de trabalho, monotonia ou ritmos intensos no serviço e até aos psicossociais por conflitos de relações. Uma longa exposição a estes fatores pode causar o surgimento de doenças ou acidentes relacionados ao trabalho.

Segundo a Organização Internacional do Trabalho (OIT), ocorrem anualmente 2,34 milhões de mortes decorrentes de acidentes ou doenças ocasionadas no trabalho. Destas, cerca de 6.300 mortes acontecem em seus ambientes de trabalho e 5.500 são causadas por doenças decorrentes de suas práticas profissionais (OIT, 2013).

A ST, de acordo com o MTE, teve um avanço ao tratar da segurança nos serviços de saúde, bem como daqueles que exercem atividades de promoção e assistência ao bem-estar em geral. A NR 32 determina diretrizes voltadas para a implementação de técnicas de proteção à saúde e segurança do trabalhador (Brasil, 2005).

Para os trabalhadores de saúde, a NR 32 foi fundamental, pois a perda da capacidade laboral e o afastamento de pessoal são consequências das condições insatisfatórias dos locais de trabalho. A adesão à esta Norma proporciona melhorias para a saúde e segurança dos prestadores de serviço, diminuindo doenças e agravos, reduzindo as taxas de absenteísmo decorrentes de acidentes ocupacionais e favorecendo o aumento de produtividade. Para a implantação da Norma são necessários recursos físicos e materiais, além de preparação específica e motivação dos funcionários e gestores para a obtenção de novos comportamentos e hábitos (Santos et al., 2012).

A Ergonomia merece destaque. De acordo com o Global Burden of Disease (GBD), sem a otimização das condições de trabalho, as condições musculoesqueléticas são as que mais contribuíram para uma vida das pessoas com dor, atingindo cerca de $16 \%$ de todos os anos vividos com deficiência (GBD, 2019).

Diante do exposto, por meio da Ergonomia é possível colaborar para mudanças nas condições e no ambiente laboral, mediante adaptações nos recursos e no processo de trabalho para melhorar a qualidade de vida dos empregados. A Ergonomia também se volta à prevenção dos danos causados aos trabalhadores durante o desempenho de suas funções, de modo a reduzir riscos, sem prejuízo ao desempenho profissional e até mesmo individual (Santos et al., 2021). Por sua vez, o ambiente laboral 
abrange um conjunto de fatores que causam riscos ao profissional, comprometendo sua saúde a curto ou longo prazo (Silva et al., 2021).

O ambiente físico de trabalho incluindo estrutura física, ar, maquinário, móveis, produtos, substâncias químicas, materiais e os processos de produção no local de trabalho, podem afetar a segurança e saúde física dos trabalhadores, bem como sua saúde mental e seu bem-estar. Os perigos no ambiente físico normalmente podem incapacitar ou até mesmo causar a morte dos trabalhadores. Essa seria a razão para que as primeiras leis e normas de segurança e saúde dos trabalhadores focalizassem esses fatores. Ainda hoje, tais perigos continuam ameaçando as vidas dos trabalhadores diariamente, tanto em países desenvolvidos como em países em desenvolvimento (OMS, 2010). Diante da relevância do tema, procurou-se trazê-lo para o cenário das CMEs, espaço de cuidado e de processamento de produtos para a saúde estéreis e seguros e que, paradoxalmente, tantos riscos oferece aos seus trabalhadores. Este estudo objetiva analisar as produções científicas sobre riscos ergonômicos na CME.

\section{Metodologia}

Trata-se de estudo de revisão integrativa da literatura, que possibilita a síntese e a conclusão geral do conhecimento científico de um determinado tema específico. Os componentes da revisão integrativa percorrem seis etapas: identificação do tema e seleção da hipótese ou questão de pesquisa; estabelecimento de critérios para inclusão e exclusão de estudos/amostragem ou busca na literatura; definição das informações a serem extraídas dos estudos selecionados/categorização dos estudos; avaliação dos estudos incluídos na revisão integrativa; interpretação dos resultados e apresentação da revisão/síntese do conhecimento (Mendes, Campos, \& Galvão, 2008).

As questões norteadoras elaboradas foram: Quais as fontes de riscos ergonômicos para trabalhadores de enfermagem nas CMEs? Quais seriam as estratégias para reduzir riscos ergonômicos nas CMEs? Que consequências trariam as falhas na prevenção dos riscos ergonômicos nas CMEs?

Os principais assuntos foram identificados e sistematizados pelos elementos Participante, Interesse e Contexto, conhecido como estratégia PICo (Quadro 1), que é um mnemônico adaptado pelo Joanna Briggs Institute.

Quadro 1: Aplicação da estratégia PICO.

\begin{tabular}{|c|c|l|}
\hline ACRÔNIMO & DEFINIÇÃO & \multicolumn{1}{|c|}{ APLICAÇÃO } \\
\hline P & Participante & Riscos Ergonômicos \\
\hline I & Interesse & Análise dos riscos ergonômicos no setor e estratégias para reduzi-los \\
\hline Co & Contexto & Central de Material e Esterilização \\
\hline
\end{tabular}

Fonte: Autores (2021).

Para a seleção dos artigos foram utilizados como critérios de inclusão: artigos disponíveis nos idiomas português, inglês e espanhol, na forma do artigo científico, com texto completo. As exclusões abrangeram livros e estudos que tratavam de CMEs terceirizadas, já que nelas não se conhecia o cenário de risco para seus trabalhadores. Durante a busca, o tema foi obtido em poucos estudos, levando os autores a não considerar inicialmente o ano de publicação dos mesmos.

A busca bibliográfica ocorreu no período de abril a maio de 2021. Para a construção das estratégias de busca foram utilizados nos termos as aspas (") para restringir termos compostos e estabelecer sua ordem. Os operadores booleanos e/ou de pesquisa AND - interseção dos termos, OR — união dos termos compostos e/ou agrupamento dos sinônimos também foram empregados. Foram consultadas as Bases de Dados Sistema Online de Busca e Análise de Literatura Médica 
(MEDLINE)/PubMed, Literatura Latino-Americana e do Caribe em Ciências da Saúde (Lilacs) e Base de Dados em Enfermagem (BDEnf) da Biblioteca Virtual em Saúde (BVS). Foi realizada ainda a busca manual para obtenção de outros estudos, favorecendo uma seleção mais ampla.

O conjunto de referências identificadas nas bases de dados foi exportado e organizado em uma planilha Excel para análise e seleção pelo autor, título, ano, volume, número, página, base de dados, resumo e tipo de artigo.

Os descritores para realizar a busca foram aqueles contemplados nos Descritores em Ciências da Saúde (DeCS). A Tabela 1 apresenta as estratégias de buscas.

Tabela 1: Estratégias de buscas realizadas em 2021.

\begin{tabular}{|c|c|c|}
\hline BASES & ESTRATÉGIAS & $\mathbf{N}$ \\
\hline BDENF & $\begin{array}{l}\text { "ergonomia" OR “ergonomics” OR “ergonomía” AND “enfermagem” OR “nursing” OR “enfermería” AND } \\
\text { "esterilização" OR "sterilization" OR "esterilizacíon” }\end{array}$ & 2 \\
\hline BDENF & $\begin{array}{l}\text { "ergonomia" OR "ergonomics" OR "ergonomía” AND “saúde do trabalhador" OR "occupational health" OR "salud } \\
\text { laboral” AND "esterilização" OR "sterilization" OR "esterilizacíon" }\end{array}$ & 17 \\
\hline BDENF & $\begin{array}{l}\text { "ergonomia" OR "ergonomics" OR "ergonomía” AND "enfermagem" OR "nursing” OR “enfermería"AND "saúde } \\
\text { do trabalhador" OR "occupational health" OR "salud laboral" }\end{array}$ & 3 \\
\hline BDENF & $\begin{array}{l}\text { "ergonomia" OR "ergonomics" OR "ergonomía" AND "enfermagem" OR "nursing” OR "enfermería” AND "saúde } \\
\text { do trabalhador" OR "occupational health" OR "salud laboral" AND "programa de saúde ocupacional" OR } \\
\text { "occupational health program" OR "programa de salud laboral" }\end{array}$ & 1 \\
\hline BDENF & $\begin{array}{l}\text { "recursos humanos de enfermagem" OR "nursing Staff” OR "personal de enfermería" AND "saúde do trabalhador" } \\
\text { OR "occupational health" OR "salud laboral" AND "riscos ocupacionais" OR "occupational risks" OR "riesgos } \\
\text { laborales" AND "esterilização" OR "sterilization" OR "esterilización" }\end{array}$ & 29 \\
\hline BDENF & $\begin{array}{l}\text { "enfermagem" OR "nursing" OR “enfermería” AND "riscos ocupacionais" OR “occupational risks" OR "riesgos } \\
\text { laborales" AND "esterilização" OR "sterilization" OR "esterilización” AND "ergonomia"OR "ergonomics" OR } \\
\text { "ergonomía" }\end{array}$ & 9 \\
\hline LILACS & $\begin{array}{l}\text { "ergonomia" OR “ergonomics" OR “ergonomía” AND "enfermagem” OR "nursing” OR "enfermería” AND } \\
\text { "esterilização" OR "sterilization" OR "esterilizacíon" }\end{array}$ & 3 \\
\hline LILACS & 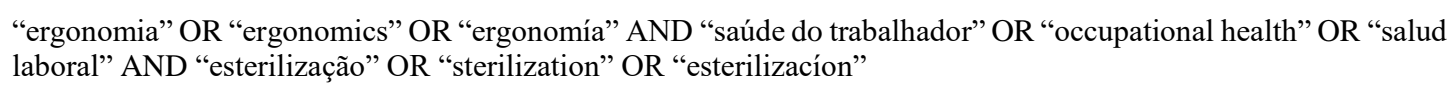 & 109 \\
\hline LILACS & $\begin{array}{l}\text { "ergonomia" OR "ergonomics" OR "ergonomía" AND "saúde do trabalhador" OR "occupational health" OR "salud } \\
\text { laboral" AND "licença médica" OR "sick leave" OR "ausencia por enfermedad" AND "esterilização" OR } \\
\text { "sterilization" OR "esterilizacíon" }\end{array}$ & 1 \\
\hline LILACS & $\begin{array}{l}\text { "ergonomia" OR “ergonomics" OR “ergonomía” AND “enfermagem” OR “nursing” OR “enfermería”AND "saúde } \\
\text { do trabalhador" OR “occupational health" OR "salud laboral” }\end{array}$ & 2 \\
\hline LILACS & $\begin{array}{l}\text { "recursos humanos de enfermagem" OR "nursing Staff" OR "personal de enfermería" AND "saúde do trabalhador" } \\
\text { OR "occupational health" OR "salud laboral" AND "riscos ocupacionais" OR "occupational risks" OR "riesgos } \\
\text { laborales" AND "esterilização" OR "sterilization" OR "esterilización" }\end{array}$ & 30 \\
\hline LILACS & $\begin{array}{l}\text { "enfermagem" OR "nursing” OR “enfermería" AND "riscos ocupacionais" OR “occupational risks" OR "riesgos } \\
\text { laborales" AND "esterilização" OR "sterilization" OR "esterilización” AND "ergonomia" OR "ergonomics" OR } \\
\text { "ergonomía" }\end{array}$ & 9 \\
\hline
\end{tabular}


PUBMED “ergonomia” OR “ergonomics” OR “ergonomía” AND “enfermagem” OR "nursing” OR "enfermería” AND "estratégias locais" OR "local strategies" OR "estrategias locales" AND "saúde do trabalhador" OR "occupational health" OR "salud laboral" AND "programa de saúde ocupacional" OR "occupational health program" OR "programa de salud laboral" AND "enfermagem do trabalho" OR "occupational health nursing" OR "enfermería del trabajo" AND "acidentes de trabalho" OR "accidents, occupational" OR "accidentes de trabajo" AND "licença médica" OR "sick leave" OR "ausencia por enfermedad" AND "riscos ocupacionais" OR "occupational risks" OR "riesgos laborales" AND "pesquisa" OR "research" OR "recherche" AND "saúde" OR "health" OR "salud" AND "programa de prevenção de riscos no ambiente de trabalho" OR "program of risk prevention on working environment" OR "programa de prevención de riesgos en el ambiente de trabajo" AND "regulamentação governamental" OR "government regulation OR Regulación gubernamental" AND "segurança de equipamentos" OR "equipment safety" OR "seguridad de equipos" AND "esterilização"OR "sterilization" OR "esterilizacíon" AND "materiais" OR "materials" OR "materiales" AND "recursos materiais em saúde" OR "material resources in health" OR "recursos materiales en salud"

PUBMED “ergonomia” OR “ergonomics” OR “ergonomía” AND “enfermagem” OR "nursing” OR “enfermería” AND "saúde do trabalhador" OR "occupational health" OR "salud laboral" AND "programa de saúde ocupacional" OR "occupational health program" OR "programa de salud laboral" AND "licença médica" OR "sick leave" OR "ausencia por enfermedad" AND "riscos ocupacionais" OR "occupational risks" OR "riesgos laborales" AND "regulamentação governamental" OR "government regulation" OR "regulación gubernamental" AND “esterilização" OR "sterilization" OR "esterilizacíon”AND "materiais” OR "materials” OR "materiales" AND "recursos materiais em saúde" OR "material resources in health" OR "recursos materiales en salud"

PUBMED “ergonomia” OR “ergonomics” OR “ergonomía” AND “enfermagem” OR "nursing” OR "enfermería” AND 305 "esterilização" OR "sterilization” OR “esterilizacíon”

PUBMED “ergonomia” OR “ergonomics” OR “ergonomía” AND “saúde do trabalhador” OR "occupational health” OR “salud 305 laboral" AND "esterilização" OR "sterilization" OR "esterilizacíon"

PUBMED "ergonomia” OR "ergonomics" OR "ergonomía” AND "saúde do trabalhador" OR "occupational health" OR "salud laboral" AND "licença médica" OR "sick leave" OR "ausencia por enfermedad" AND "esterilização" OR "sterilization" OR “esterilizacíon”

PUBMED “ergonomia” OR “ergonomics” OR “ergonomía” AND “enfermagem” OR “nursing” OR “enfermería” AND

"saúde do trabalhador" OR "occupational health" OR "salud laboral"

PUBMED “ergonomia” OR “ergonomics” OR “ergonomía” AND “enfermagem” OR “nursing” OR “enfermería” AND "saúde do trabalhador" OR "occupational health" OR "salud laboral” AND "esterilização" OR "sterilization" OR "esterilizacíon"

PUBMED “ergonomia” OR “ergonomics” OR “ergonomía” AND “enfermagem” OR “nursing” OR “enfermería” AND

"regulamentação governamental” OR "government regulation" OR "regulación gubernamental"

PUBMED “ergonomia” OR “ergonomics” OR “ergonomía” AND “enfermagem” OR “nursing” OR “enfermería” AND "saúde do trabalhador" OR "occupational health" OR "salud laboral" AND "programa de saúde ocupacional" OR "occupational health program" OR "programa de salud laboral"

PUBMED "recursos humanos de enfermagem" OR "nursing Staff” OR "personal de enfermería" AND "saúde do trabalhador" OR "occupational health" OR "salud laboral" AND "ergonomia" OR "ergonomics" OR "ergonomía" AND “esterilização" OR "sterilization" OR "esterilización” AND “administração de materiais no hospital" OR "materials management, hospital" OR "administración de materiales de hospital”

PUBMED "recursos humanos de enfermagem" OR "nursing Staff” OR "personal de enfermería” AND "saúde do trabalhador" OR "occupational health" OR "salud laboral" AND "riscos ocupacionais" OR "occupational risks" OR "riesgos laborales" AND "esterilização" OR "sterilization" OR "esterilización”

PUBMED “enfermagem” OR “nursing” OR “enfermería” AND "riscos ocupacionais” OR "occupational risks” OR "riesgos laborales" AND "esterilização" OR "sterilization" OR "esterilización” AND “ergonomia”OR "ergonomics" OR "ergonomía"

Fonte: Autores (2021)

O estudo atendeu as etapas de elaboração recomendadas pelo Preferred Reporting Items for Systematic Reviews and Meta-Analyses (PRISMA) (Joanna Briggs Institute, 2014). O processo de identificação, seleção, elegibilidade e inclusão está detalhado no fluxograma abaixo (Figura 1). 
Figura 1: Fluxograma do processo de seleção dos estudos primários.

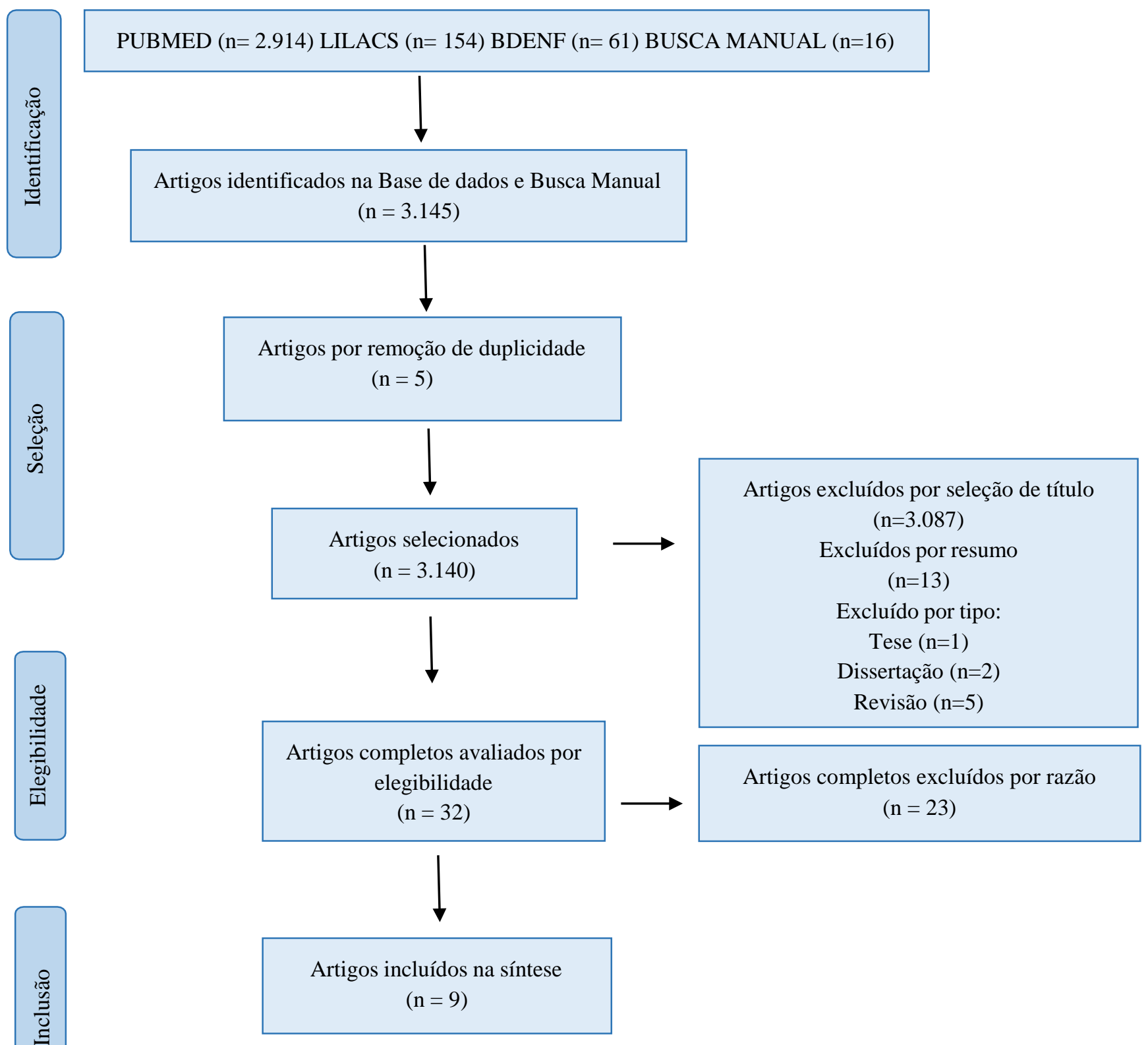

Fonte: Autores (2021).

\section{Resultados e Discussão}

Os resultados aqui apresentados reúnem os achados de 9 estudos. O fluxograma PRISMA apresentado na figura 1 sumariza as buscas e exclusões durante o processo de seleção. Foram excluídos 5 artigos por duplicidade, 3087 por seleção de título, 13 pelo resumo, e 8 excluídos pelo tipo (tese $n=1$, dissertação $n=2$, revisão $=5$ ). Restaram para a análise de texto completo 32 artigos. Após houve leitura detalhada dos títulos e resumos para análise qualitativa. 23 artigos foram excluídos por não atenderem ao escopo do estudo. Dessa forma, 9 artigos foram incluídos na revisão integrativa.

O descritor Esterilização motivou a exclusão de 3.087 estudos que envolviam bancários, costureiras, pescadores, além da esterilização humana. Foi realizada também a busca manual de estudos para ampliar a análise da temática.

Nesta revisão prevaleceram as revistas brasileiras $(n=8)$. Somente um artigo foi publicado em revista americana (n=1). Foram distribuídos nos anos a partir de 2013 (n=1), 2014 (n=2), $2015(n=1), 2016(n=1), 2017$ (n=2), 2018 (n=2). 
Não houve predominância de autores.

Os estudos selecionados ouviram um mínimo de 12 e o máximo de 77 profissionais de enfermagem. Instituições públicas e privadas foram contempladas.

Estudos envolvendo CME's terceirizadas foram excluídos, pela impossibilidade de avaliação dos riscos ergonômicos descritos por estarem fora do contexto do hospital.

No Quadro 2 estão descritos os estudos que compuseram a amostra, apresentando: área temática, periódico, ano, país, tipo de estudo, autores, título e a síntese dos resultados.

Quadro 2: Caracterização dos estudos investigados.

\begin{tabular}{|c|c|c|c|c|c|}
\hline $\mathbf{N}$ & $\begin{array}{c}\text { ÁREA } \\
\text { TEMÁTICA }\end{array}$ & $\begin{array}{c}\text { PERIÓDICO } \\
\text { ANO } \\
\text { PAÍS } \\
\text { TIPO DE ESTUDO }\end{array}$ & AUTORES & TÍTULO & SÍNTESE DOS RESULTADOS \\
\hline 1 & $\begin{array}{c}\text { Riscos } \\
\text { ergonômicos } \\
\text { na } \\
\text { enfermagem } \\
\text { na CME }\end{array}$ & $\begin{array}{l}\text { Revista Cuidarte } \\
\qquad 2018 \\
\text { Brasil } \\
\text { Estudo exploratório } \\
\text { e quantitativo }\end{array}$ & $\begin{array}{l}\text { Lima, } \\
\text { Chaves, } \\
\text { Lima, } \\
\text { Silva, } \\
\text { Soares, \& } \\
\text { Santos }\end{array}$ & $\begin{array}{c}\text { Riscos } \\
\text { ocupacionais em } \\
\text { profissionais de } \\
\text { enfermagem de } \\
\text { centros de } \\
\text { material e } \\
\text { esterilização. }\end{array}$ & $\begin{array}{l}\text { Objetivo: Identificar os riscos ocupacionais em } \\
\text { profissionais de Enfermagem de CME. } 77 \\
\text { profissionais. Riscos ergonômicos: posturas } \\
\text { forçadas (90\%); Riscos biológicos: } \\
\text { vulnerabilidade às infecções (95\%); Riscos } \\
\text { Físicos: ruídos fortes (93\%); temperatura } \\
\text { inapropriada }(85 \%) \text {; Riscos químicos: gases e } \\
\text { aerossóis }(65 \%) \text {. }\end{array}$ \\
\hline 2 & $\begin{array}{c}\text { Riscos } \\
\text { ergonômicos } \\
\text { na } \\
\text { enfermagem } \\
\text { na CME }\end{array}$ & $\begin{array}{c}\text { Rev. Enferm. UFPI } \\
2016 \\
\text { Brasil } \\
\begin{array}{c}\text { Estudo descritivo, } \\
\text { transversal }\end{array}\end{array}$ & $\begin{array}{l}\text { Gouveia, } \\
\text { Oliveira, \& } \\
\text { Lira }\end{array}$ & $\begin{array}{c}\text { Riscos } \\
\text { ergonômicos em } \\
\text { um centro de } \\
\text { material e } \\
\text { esterilização. }\end{array}$ & $\begin{array}{l}\text { Objetivo: Identificar os fatores de risco } \\
\text { ergonômicos para profissionais de enfermagem no } \\
\text { CME. } 48 \text { profissionais. Fatores de Riscos } \\
\text { Ergonômicos: desenho arquitetônico dos postos de } \\
\text { trabalho }(58,3 \%) \text {, distribuição inadequada de } \\
\text { pessoal e/ou equipe }(79,2 \%) \text {, ferramentas } \\
\text { insuficientes ou inadequadas de trabalho }(79,2 \%) \text {, } \\
\text { manutenção excessiva de uma mesma postura no } \\
\text { trabalho }(58,3 \%) \text {. Necessidade de adotar posturas } \\
\text { forçadas nãoconfortáveis }(83,3 \%) \text {. Repetitividade } \\
\text { excessiva de movimentos }(91,7 \%) \text {, manejo de } \\
\text { cargas (70,8\%), risco de contaminação externa } \\
(91,7 \%) \text { e conhecimento ergonômico insuficiente } \\
(68,7 \%) \text {. }\end{array}$ \\
\hline 3 & $\begin{array}{c}\text { Riscos } \\
\text { ergonômicos } \\
\text { na } \\
\text { enfermagem }\end{array}$ & $\begin{array}{c}\text { Workplace health \& } \\
\text { safety } \\
2013 \\
\text { Estados Unidos } \\
\text { Estudo de campo }\end{array}$ & $\begin{array}{l}\text { Rogers, } \\
\text { Buckheit, \& } \\
\text { Ostenford }\end{array}$ & $\begin{array}{l}\text { Ergonomics and } \\
\text { nursing in hospital } \\
\text { environments. }\end{array}$ & $\begin{array}{l}\text { Objetivo: Descrever as condições de trabalho, o } \\
\text { ambiente e as atividades que podem contribuir } \\
\text { para lesões musculoesqueléticas. Participantes: } 42 \\
\text { enfermeiros. Cinco hospitais. Grupos focais, } \\
\text { entrevistas e observação. Sugestões: } \\
\text { redesenho dos ambientes de trabalho com a } \\
\text { participação de enfermeiros; novos } \\
\text { equipamentos e insumos; aumento do staff } \\
\text { especialmente masculino; oferta de programas de } \\
\text { educação em horários convenientes para todos os } \\
\text { turnos; descontos para a aquisição de sapatos } \\
\text { e meias de compressão. }\end{array}$ \\
\hline
\end{tabular}




\begin{tabular}{|c|c|c|c|c|c|}
\hline 4 & $\begin{array}{c}\text { Ergonomia } \\
\text { biomecânica } \\
\text { na } \\
\text { enfermagem }\end{array}$ & $\begin{array}{l}\text { Perspectivas Online: } \\
\text { Biológicas e Saúde } \\
\qquad 2017 \\
\text { Brasil } \\
\text { Estudo } \\
\text { exploratório, } \\
\text { descritivo, } \\
\text { quantitativo }\end{array}$ & $\begin{array}{l}\text { Freire, } \\
\text { Soares, \& } \\
\text { Torres }\end{array}$ & $\begin{array}{c}\text { Influência da } \\
\text { ergonomia na } \\
\text { biomecânica de } \\
\text { profissionais de } \\
\text { enfermagem no } \\
\text { ambiente } \\
\text { hospitalar. }\end{array}$ & $\begin{array}{l}\text { Objetivo: Identificar a ocorrência de } \\
\text { lombalgia em profissionais de enfermagem, } \\
\text { correlacionar a incidência com aaplicabilidade dos } \\
\text { princípios ergonômicos e divulgar tais princípios } \\
\text { e sua aplicabilidade para a equipe de enfermagem. } \\
20 \text { profissionais da equipe de enfermagem. Setor } \\
\text { de clínica médica. Hospital geral. Incidência de } \\
\text { lombalgia durante as atividades: } 80 \% \text {; após a } \\
\text { jornada de trabalho: } 75 \% \text {. } 65 \% \text { dos profissionais } \\
\text { sem orientações ergonômicas, resultando na } \\
\text { aplicabilidade insuficiente de tais princípios. }\end{array}$ \\
\hline 5 & $\begin{array}{c}\text { Riscos } \\
\text { ocupacionais } \\
\text { na CME }\end{array}$ & $\begin{array}{l}\text { SOBECC } \\
2014 \\
\text { Brasil } \\
\text { Estudo } \\
\text { exploratório, } \\
\text { transversal, } \\
\text { quantitativo }\end{array}$ & $\begin{array}{l}\text { Aquino, } \\
\text { Barros, } \\
\text { Brito, } \\
\text { Ferreira, } \\
\text { Medeiros, } \\
\text { \& Santos }\end{array}$ & $\begin{array}{l}\text { Centro de } \\
\text { material e } \\
\text { esterilização: } \\
\text { acidentes de } \\
\text { trabalho e riscos } \\
\text { ocupacionais. }\end{array}$ & $\begin{array}{l}\text { Objetivo: Investigar a ocorrência de acidentes de } \\
\text { trabalho e os principais riscos ocupacionais a que } \\
\text { estão expostos os profissionais de Enfermagem } \\
\text { em CME. } 37 \text { profissionais. } 27,3 \% \text { dos } \\
\text { profissionais da instituição pública já sofreram } \\
\text { acidentes; } 83,3 \% \text { do tipo perfurocortante. } \\
\text { Instituição privada, } 46,7 \% \text {, sendo } 57,1 \% \text { por } \\
\text { queimaduras na autoclave. }\end{array}$ \\
\hline 6 & $\begin{array}{c}\text { Riscos } \\
\text { ergonômicos } \\
\text { no CC }\end{array}$ & $\begin{array}{c}\text { Revista } \\
\text { Enfermagem } \\
\text { Contemporânea } \\
2017 \\
\text { Brasil } \\
\text { Estudo } \\
\text { exploratório } \\
\text { quanti- } \\
\text { qualitativa }\end{array}$ & $\begin{array}{l}\text { Brito \& } \\
\text { Correio }\end{array}$ & $\begin{array}{l}\text { Caracterização do } \\
\text { desconforto físico } \\
\text { relacionado à } \\
\text { ergonomia em } \\
\text { profissionais de } \\
\text { enfermagem do } \\
\text { centro cirúrgico. }\end{array}$ & $\begin{array}{l}\text { Objetivos: Caracterizar os profissionais de } \\
\text { enfermagem do centro cirúrgico, identificar as } \\
\text { queixas relacionadas ao desconforto físico } \\
\text { decorrentes de riscos ergonômicos; sua } \\
\text { localização anatômica, caracterizar e correlacionar } \\
\text { as queixas com o ambiente de trabalho, verificar o } \\
\text { tratamento; detectar as possíveis intervenções } \\
\text { realizadas para prevenção dos riscos ergonômicos. } \\
22 \text { profissionais de enfermagem. } 68,18 \% \text { dos } \\
\text { participantes desconforto nos membros } \\
\text { superiores, coluna ou nos membros inferiores. } \\
\text { Locais mais doloridos: ombros }(18,91 \%) \text {, coluna } \\
(18,91 \%) \text { e pernas }(18,91 \%) \text { Queixas: } \\
\text { dor (40,74\%), dolorimento }(22,22 \%) \text { e cansaço } \\
(18,51 \%) .86,36 \% \text { participantes informou que a } \\
\text { unidade em que atuam não promove alguma } \\
\text { intervenção para prevenção ou tratamento dos } \\
\text { riscos ergonômicos. } 63,63 \% \text { participantes } \\
\text { desconfortos relacionados ao trabalho no setor } \\
\text { atual. }\end{array}$ \\
\hline 7 & $\begin{array}{l}\text { Visibilidade do } \\
\text { enfermeiro da } \\
\text { CME }\end{array}$ & $\begin{array}{l}\text { Texto e Contexto } \\
\text { Enfermagem } \\
2018 \\
\text { Brasil } \\
\text { Estudo } \\
\text { exploratório, } \\
\text { descritivo }\end{array}$ & $\begin{array}{l}\text { Sanchez, } \\
\text { Silveira, } \\
\text { Figueiredo, } \\
\text { Mancia, } \\
\text { Schwonke, \& } \\
\text { Gonçalves }\end{array}$ & $\begin{array}{l}\text { Estratégias que } \\
\text { contribuem paraa } \\
\text { visibilidade do } \\
\text { trabalho do } \\
\text { enfermeiro na } \\
\text { central de } \\
\text { material e } \\
\text { esterilização. }\end{array}$ & $\begin{array}{l}\text { Objetivo: Identificar estratégias para promover o } \\
\text { reconhecimento e a visibilidade do trabalho do } \\
\text { enfermeiro na CME. } 17 \text { participantes. Resultados: } \\
\text { destaque para a troca de vivência entre } \\
\text { enfermeiros da CME e de outras unidades; seleção } \\
\text { dos trabalhadores, a partir de critérios necessários } \\
\text { para a atuação no local; educação Permanente, } \\
\text { com temáticas envolvendo CME. }\end{array}$ \\
\hline 8 & $\begin{array}{c}\text { Riscos } \\
\text { ergonômicos } \\
\text { na } \\
\text { enfermagem }\end{array}$ & $\begin{array}{l}\text { Rev. Enferm. UFPE } \\
\text { on line } \\
2014 \\
\text { Brasil } \\
\text { Estudo } \\
\text { exploratório, } \\
\text { descritivo, } \\
\text { qualitativo }\end{array}$ & $\begin{array}{l}\text { Oliveira, } \\
\text { Guimaraes, } \\
\text { Da Cruz } \\
\text { Silva, Silva, } \\
\text { de Sousa, \& } \\
\text { Moura }\end{array}$ & $\begin{array}{c}\text { Saúde e } \\
\text { segurança do } \\
\text { enfermeiro: riscos } \\
\text { ergonômicos nos } \\
\text { setores críticos do } \\
\text { ambiente } \\
\text { hospitalar. }\end{array}$ & $\begin{array}{l}\text { Objetivo: Analisar riscos ergonômicos de } \\
\text { enfermeiros de áreas críticas do hospital; } \\
\text { identificando fatores de riscos a eles associados. } \\
27 \text { enfermeiros. Resultados: Riscos ergonômicos: } \\
\text { ambiente de trabalho e desenvolvimento do risco } \\
\text { ergonômico; tempo de exposição do enfermeiro } \\
\text { aos riscos ergonômicos. Riscos ergonômicos } \\
\text { identificados: esforço físico, postura inadequada, } \\
\text { levantamento e transporte manual de peso, } \\
\text { trabalho diuturno e repetitividade. }\end{array}$ \\
\hline
\end{tabular}




\begin{tabular}{|c|c|c|c|c|c|}
\hline 9 & $\begin{array}{c}\text { Riscos } \\
\text { ocupacionais } \\
\text { na CME }\end{array}$ & $\begin{array}{l}\text { Rev. Min. Enferm. } \\
\qquad \begin{array}{c}2015 \\
\text { Brasil } \\
\text { Estudo } \\
\text { qualitativo }\end{array}\end{array}$ & $\begin{array}{c}\text { Bittencourt, } \\
\text { Benetti, } \\
\text { Graube, } \\
\text { Stumm, \& } \\
\text { Kaiser }\end{array}$ & $\begin{array}{l}\text { Vivências de } \\
\text { profissionais de } \\
\text { enfermagem sobre } \\
\text { riscos ambientais } \\
\text { em um centro de } \\
\text { material e } \\
\text { esterilização }\end{array}$ & $\begin{array}{l}\text { Objetivo: Buscar vivências de profissionais de } \\
\text { enfermagem sobre exposição aos riscos } \\
\text { ambientais em CME. } 12 \text { profissionais. Resultado: } \\
\text { destaque para atividades que demandam esforço e } \\
\text { mobilização do corpo em condições pouco } \\
\text { apropriadas, ritmo excessivo de trabalho, postura } \\
\text { inadequada e cargas diversas. Riscos ambientais: } \\
\text { níveis de ruídos potencialmente danosos, } \\
\text { temperaturas elevadas. Riscos químicos: } \\
\text { exposição aos produtos de limpeza. }\end{array}$ \\
\hline
\end{tabular}

Fonte: Autores (2021).

A análise dos estudos selecionados foi distribuída em quatro categorias. A primeira envolveu os riscos do trabalho na CME. A segunda, comparou esses riscos aos do Centro Cirúrgico (CC), Terapia Intensiva (UTI) e Clínica Médica (CM). Na terceira analisa-se a profissão da enfermagem como um todo a partir de fatores intervenientes para riscos e que interferem diretamente no cotidiano de trabalho desses profissionais. A última categoria sinaliza uma possibilidade de mudança para o cenário da CME, com ganho da sua visibilidade no contexto hospitalar.

Na categoria 1, os riscos do trabalho em CME foram abordados diretamente em quatro estudos (Lima et al., 2018; Gouveia, Oliveira, \& Lira, 2016; Aquino et al., 2014; Bittencourt, Benetti, Graube, Stumm, \& Kaiser, 2015). Tais trabalhos trataram especificamente dos riscos ergonômicos na CME e foram publicados no intervalo de até três anos (2015 a 2018), revelando provavelmente uma realidade semelhante aos tempos atuais.

Posturas forçadas foram apontadas com percentuais que variaram entre $90 \%$ e $83,3 \%$. Os riscos ergonômicos estão presentes em dois estudos (Lima et al., 2018; Gouveia et al., 2016). Estudo que ouviu 48 profissionais de enfermagem destaca riscos e inúmeros problemas: distribuição inadequada de pessoal/ou equipe (79,2\%); ferramentas insuficientes ou inadequadas de trabalho (79,2\%); manejo de cargas (70,8\%), conhecimento ergonômico insuficiente $(68,7 \%)$, manutenção excessiva de uma mesma postura no trabalho $(58,3 \%)$ o desenho arquitetônico dos postos de trabalho (58,3\%). Esse mesmo grupo considerou como os piores problemas ergonômicos a repetitividade dos movimentos e o risco de contaminação externa $(91,7 \%)$ (Gouveia et al., 2016).

As falhas no desenho arquitetônico do posto de trabalho da enfermagem na CME tornam o local inadequado e criam problemas. Podem ser citados: cruzamento de material limpo com o material estéril; local de entrega de material, reforma no expurgo, área estreita para as autoclaves (dificultando ou impossibilitando a circulação de carrinhos de transporte) e armazenamento de material; além das mobílias e máquinas que não seguem os princípios ergonômicos (Gouveia et al., 2016).

As ferramentas de trabalho consideradas insuficientes ou inadequadas foram exemplificadas por carrinhos quebradose em alturas inadequadas para as autoclaves, necessitando de maior esforço para a colocação e/ou retirada das cargas nas autoclaves, levando à postura forçada dos funcionários durante expediente (Gouveia et al., 2016).

Essas pesquisas ainda destacaram outros riscos presentes na CME como os biológicos, exemplificados pela vulnerabilidade às infecções (95\%); os físicos incluindo ruídos fortes (93\%), temperatura inapropriada (85\%); os químicos abrangendo gases e aerossóis (65\%) (Lima et al., 2018).

Profissionais da CME relataram não ser fácil realizar o armazenamento dos instrumentos em prateleiras e fazer suas entregas a outros setores. $\mathrm{O}$ fato de muitos materiais serem pesados pode prejudicar à saúde. O esforço físico é bastante presente assim como a repetição do processo várias vezes ao dia. Essa repetição se aplica tanto na atividade de empurrar e puxar carrinhos e racks; no transporte de materiais além da questão de abrir e fechar as autoclaves. Esses foram exemplos dos riscos relacionados a esses profissionais executando tais tarefas (Bittencourt et al., 2015). 
Para Lima et. al. (2018), os fatores extrínsecos, ligados às condições que facilitam o desgaste físico, e não relacionados ao trabalho, também podem favorecer o desenvolvimento e a o agravo da lombalgia e outras doenças osteomusculares. Enfatiza-se ao enfermeiro gestor e a equipe de enfermagem, adotar novas estratégias para evitar riscos ocupacionais ocasionados no ambiente de trabalho da CME. A estratégia de prevenção de agravos à saúde sugerida foi o incentivo ao uso adequado de Equipamento de Proteção Individual (EPIs) pela gestão.

Um dos estudos sobre riscos ambientais na CME, incluiu agentes físicos, químicos, biológicos e ergonômicos. Com relação à Ergonomia, foi destacado que para trabalhar na CME e executar devidamente as funções, é necessário esforço físico (Bittencourt et al., 2015).

A categoria 2, trata dos riscos ergonômicos em outros setores do hospital buscando estabelecer similaridades e diferenças com os riscos relativos à CME. Foram relacionados estudos nos cenários de centro cirúrgico, clínica médica, terapia intensiva e hemodiálise.

A análise do desconforto de profissionais de enfermagem de centro cirúrgico relacionada à Ergonomia evidenciou problemas nos membros superiores, coluna ou membros inferiores, evidenciando risco potencial para problemas osteomusculares. Nesse estudo realizado no Brasil, o grupo de 22 profissionais ouvidos destaca uma série de desconfortos como dor e cansaço. A maior parte dos participantes $(86,36 \%)$ informou que a unidade de CC não promove intervenção para prevenção ou tratamento dos riscos ergonômicos. Os participantes informaram que sentem dor, fadiga, desconforto muscular, insônia (Brito \& Correio, 2017).

A lombalgia também está presente nos trabalhadores da clínica médica. Estudo que analisa o ponto e vista e a experiência de 20 profissionais desse setor revela que grande parte deles sente dores durante a realização das atividades laborativas e também no seu período de descanso e folgas. Ao serem questionados sobre a percepção da relação da dor com o trabalho, (80\%) entendem que a causa da dor lombar está ligada à atividade laborativa (Freire, Soares, \& Torres, 2017).

Os riscos ergonômicos nos setores críticos do ambiente hospitalar como centro cirúrgico (CC), unidade de tratamento (UTI) e hemodiálise podem ser relacionados ao ritmo excessivo de trabalho, e trabalho nos turnos diurno e noturno. As justificativas mencionadas para os problemas levantados incluem a insatisfação salarial, a deficiência de recursos humanos na instituição. As posturas inadequadas, ações repetitivas, levantamento manual de peso e estresse físico e psíquico, se dão por ambiente de trabalho inadequado. Esse grupo de 27 profissionais destacou também o elevado esforço físico, as longas jornadas de trabalho, o uso de máquinas e equipamentos malconservados. Os agentes causais responsáveis por postura inadequada foram mobiliários necessitando de manutenção, poltronas dos pacientes baixas e até iluminação inadequada (Oliveira et al., 2014).

É fácil constatar a similaridade de problemas e agentes responsáveis pelos riscos ergonômicos e desconfortos trazidos para os profissionais de enfermagem na CME ou nos setores críticos do hospital.

A categoria 3 verifica se o trabalho da enfermagem na CME estaria fora do contexto de riscos ergonômicos quando comparado aos demais profissionais. Os estudos selecionados confirmam que não.

Os profissionais de enfermagem se submetem a dupla ou tripla jornada de trabalho, o que os expõe a riscos de acidentes relacionados a desgaste físico e emocional. Os acidentes de trabalho acontecem com maior probabilidade durante os plantões diurnos devido a demanda de atividades. As doenças ocupacionais são destacadas e relacionadas com atividadesque necessitam grande esforço físico, com ritmo ou intensidade alta, e em condições não ergonômicas. Estão incluídas a fadiga muscular, varizes, estresse, problemas oculares, lombalgias, transtornos de sono e as lesões na coluna vertebral; responsáveis pelos danos físicos, em virtude da falta de conhecimento sobre medidas preventivas (Aquino et al., 2014).

Já na jornada de trabalho diurna, o movimento cirúrgico intenso demanda materiais prontos imediatamente para 
uso, exigindo agilidade e tarefas sem interrupções dos funcionários. O preparo dos materiais após os procedimentos cirúrgicos acaba sendo realizado nos turnos diurno e noturno continuamente (Gouveia et al., 2016). Uma boa parte dos profissionais de enfermagem possui outro vínculo empregatício, podendo ser justificada a dupla jornada pela melhoria financeira para suprir as necessidades, com o risco da sobrecarga física, psíquica ou agravos pelas condições do próprio ambiente de trabalho (Lima et. al., 2018). Os riscos ergonômicos apontados foram o trabalho repetitivo, o cansaço físico e a sobrecarga de trabalho, pressão e rapidez para execução de tarefas de acordo com a demanda exigida sendo relacionado até o motivo de insatisfação dos profissionais em atuar nas suas atividades na CME (Gouveia et al., 2016; Lima et al., 2018).

Estudo sobre Ergonomia em ambiente hospitalar, enumera as atividades que podem contribuir para o surgimento de lesões musculoesqueléticas em enfermeiros como organização das estações de trabalho, carga de trabalho pesada, pessoal inadequado, falta de educação, levantamento inadequado de pacientes, falta de dispositivos auxiliares. Os participantes sugeriram o redesenho dos ambientes de trabalho com a participação de enfermeiros; novos equipamentos e insumos; aumento do staff especialmente masculino; oferta de programas de educação em horários convenientes para todos os turnos; descontos para a aquisição de sapatos e meias de compressão (Rogers, Buckheit, \& Ostenford, 2013).

Nos estudos selecionados, foram abordados os riscos ergonômicos relacionados à jornada de trabalho dupla, turnos de trabalho exaustivos, movimentos repetitivos, esforço físico, postura errada, mobiliário inadequado (Aquino et al., 2014; Gouveia et al., 2016; Lima et al., 2018).

Na categoria 4, a prática ergonômica, na CME, minimizaria esses riscos: mobiliário adequado, pausa para descanso, melhor distribuição do pessoal. O conhecimento do gestor na ergonomia poderá favorecer o interesse da instituição em oferecer treinamento para aos seus funcionários sobre o tema.

Medidas de prevenção ao risco ergonômico levam ao avanço de doenças. Os profissionais de enfermagem reconhecem a necessidade de funcionários capacitados na CME, para evitar a falta de informação e não influenciar na dinâmica interna da CME e relações interpessoais no trabalho (Bittencourt et al., 2015).

O estudo sobre Ergonomia em ambiente hospitalar revelou que 71,5\% da equipe de enfermagem é acometida pela lombalgia. Diferentes fatores predispõem a esse risco: esforço físico, postura inadequada, levantamento e transporte manual de peso, trabalho diuturno e repetitividade. A adequação do ambiente de trabalho auxilia na ação preventiva dos riscos de dores osteomusculares. Ambientes de trabalho mal projetados, tamanho dos quartos dos pacientes inadequados e outras situações obrigam o profissional a assumir uma postura física imprópria (Rogers et al., 2013).

Foi sugerida a revisão dos turnos de trabalho, horas extras e a orientação para monitorar as condições e práticas de trabalho, visando minimizar os riscos ergonômicos. Os treinamentos e educação sobre princípios de ergonomia, operação correta de dispositivos e uso adequado de equipamentos surgem como alternativa (Rogers et al., 2013). Em outro estudo, os funcionários indicam a contratação de mais profissionais, redução das horas de trabalho, a inspeção em equipamentos e tempo para descanso durante a jornada de trabalho. A educação permanente, treinamentos com técnicas de alongamento corporal durante o expediente, reeducação postura, necessidade de adequação ergonômica do mobiliário, ou treinamentos específicos foram abordados no artigo (Brito \& Correio, 2017).

A ideia de diminuir os esforços por meio de elevadores, colocando a tecnologia a favor da Ergonomia foi apresentada. $\mathrm{O}$ aumento do espaço físico, revisão nos turnos de trabalho, horas extras, monitorização das condições e práticas de trabalho; treinamentos sobre Ergonomia, operação correta de dispositivos e usos adequados de equipamentos foram mencionados (Rogers et al., 2013).

O CC, UTI e Hemodiálise são setores críticos tais como a CME. A exposição dos trabalhadores aos riscos demonstra falta de políticas públicas na área da saúde e segurança do trabalho, pouca atenção por parte dos gestores e órgãos fiscalizadores (Oliveira et al., 2014). Outros estudos mais recentes ratificam esses problemas. As causas institucionais como 
o tempo de contratação institucional, o regime trabalhista diferenciado foram sugeridos para a promoção da saúde e prevenção dos riscos dos trabalhadores, especialmente os ergonômicos (Gouveia et al., 2016). Destaca-se também a atenção dos gestores para a necessidade da prevenção de riscos à saúde, por meio de medidas de prevenção primária como a reeducação postural ocupacional (Brito \& Correio, 2017).

Essa revisão tratou dos riscos ergonômicos na CME. Considerou-se necessário destacar a relativa falta de visibilidade desse setor que poderia levar ao adiamento ou não da resolução dos problemas envolvendo trabalhadores e o trabalho. O estudo selecionado sobre a visibilidade da CME, revela que o trabalho do enfermeiro pode se tornar pouco visível diante da ausência de conhecimento acerca do trabalho desenvolvido na CME; da pouca valorização do fazer do enfermeiro da CME, por prestar uma assistência indireta com o paciente; da ausência de critérios de seleção dos funcionários e da alocação de trabalhadores com problemas de saúde e/ou limitações físicas, bem como da rotatividade de funcionários no setor; a ausência de educação permanente voltada para as atividades desenvolvidas na CME; e a pouca divulgação do seu fazer e da sua interdependência com outros setores, ocasionando problemas internos na CME (Sanchez et al., 2018).

A visibilidade traria a possibilidade da CME de se transformar em setor com menos riscos ergonômicos, facilitandoao enfermeiro gestor a criação de ambiente de trabalho mais favorável e saudável para os profissionais de enfermagem.

\section{Conclusão}

Os estudos integrantes dessa revisão sinalizaram a CME como um setor repleto de riscos de toda ordem incluindo os ergonômicos. Esses incluem os ambientes de trabalho inadequados, a falta de insumos e equipamentos; esforço físico, postura inadequada, levantamento e transporte manual de peso, trabalho diuturno e repetitividade.

Os profissionais de enfermagem ouvidos destacaram como locais mais doloridos do corpo os ombros, coluna e pernas. As queixas dos trabalhadores incluíram dor e cansaço e a não intervenção para prevenção ou tratamento dos riscos ergonômicos.

A unidade de CME, a partir dos estudos analisados, apresenta similaridade em termos dos múltiplos fatores de riscos ergonômicos quando comparada a setores como a Unidade de Terapia Intensiva, Centro Cirúrgico e Clínica Médica.

Muitos estudos abordaram riscos ergonômicos relacionados a jornada de trabalho dupla, turnos de trabalho exaustivos, movimentos repetitivos, esforço físico, postura errada, mobiliário inadequado.

A prática ergonômica, na CME, minimizaria esses riscos através de mobiliário adequado, pausa para descanso, melhor distribuição do pessoal, gestores com conhecimento de ergonomia e educação continuada preparando os funcionários, orientando-os para o tema da ergonomia, direitos e responsabilidades.

\section{Referências}

Aquino, J. M. D., Barros, L. P., Brito, S. A., Ferreira, E. B., Medeiros, S. E. G. D., \& Santos, E. R. D. (2014). Centro de material e esterilização: acidentes de trabalho e riscos ocupacionais. Rev. SOBECC, 148-154. http://dx.doi.org/10.4322/sobecc.2014.023

Bittencourt, V. L. L., Benetti, E. R. R., Graube, S. L., Stumm, E. M. F., \& Kaiser, D. E. (2015). Vivências de profissionais de enfermagem sobre riscos ambientais em um centro de material e esterilização. Reme: Revista Mineira de Enfermagem. Belo Horizonte. 19(4), 878-884. 10.5935/1415-2762.20150067

Brasil. Ministério da Saúde (MS). Agência Nacional de Vigilância Sanitária (ANVISA). Resolução n. 15, de 15 de março de 2012. Resolução da Diretoria Colegiada (RDC). Dispõe sobre requisitos de boas práticas para o processamento de produtos para saúde e dá outras providências. Brasília, DF; 2012. https://bvsms.saude.gov.br/bvs/saudelegis/anvisa/2012/rdc0015_15_03_2012.html

Brasil. Ministério do Trabalho e Emprego (MTE). Portaria 3.214, de 08 de junho de 1978. Normas Regulamentadoras - NR 17. Diário Oficial da União,Brasília, DF, 08 jun 1978. https://www.gov.br/trabalho/pt-br/inspecao/seguranca-e-saude-no-trabalho/normas-regulamentadoras/nr-17.pdf

Brasil. Ministério do Trabalho e Emprego (MTE). Portaria $\mathrm{n}^{\circ} 485$, de 11 de novembro de 2005. Norma Regulamentadora $\mathrm{n}^{\circ}$ 32. Estabelece as diretrizes básicas para a implementação de medidas de proteção à segurança e à saúde dos trabalhadores dos serviços de saúde, bem como daqueles que exercem atividades de 
promoção e assistência à saúde em geral. Brasília, DF; 2005. http://sbbq.iq.usp.br/arquivos/seguranca/portaria485.pdf

Brito, C. F., \& Pinheiro, L. M. G. (2017). Caracterização do desconforto físico relacionado à ergonomia em profissionais de enfermagem do centro cirúrgico. Revista Enfermagem Contemporânea, 6(1), 20-29. 10.17267/2317-3378rec.v6i1.1137

Cieza, A., Causey, K., Kamenov, K., Hanson, S. W., Chatterji, S., \& Vos, T. (2020). Global estimates of the need for rehabilitation based on the Global Burden of Disease study 2019: a systematic analysis for the Global Burden of Disease Study 2019. The Lancet, 396(10267), 2006-2017. https://doi.org/10.1016/ S0140-6736(20)32340-0

Espindola, M. C. G., \& Fontana, R. T. (2012). Riscos ocupacionais e mecanismos de autocuidado do trabalhador de um centro de material e esterilização. Revista Gaúcha de Enfermagem,33, 116-123. https://doi.org/10.1590/S1983-14472012000100016

Freire, L. A., Soares, T. C. N., \& dos Santos Torres, V. P. (2017). Influência da ergonomia na biomecânica de profissionais de enfermagem no ambiente hospitalar. Biológicas \& Saúde, 7(24). 10.25242/886872420171149

Gouveia, M. T. D. O., Oliveira, V. C., \& Lira, I. M. D. S. (2016). Riscos ergonômicos em um Centro de Material e Esterilização. Rev. enferm. UFPI, 42-47. https://revistas.ufpi.br/index.php/reufpi/article/view/5427/pdf

IEA, International Ergonomics Association. (2020). What Is Ergonomics? https://iea.cc/what-is-ergonomics/

Joanna Briggs Institute. (2014). Joanna Briggs Institute reviewers' manual: 2014 edition. Australia: The Joanna Briggs Institute. https://nursing.lsuhsc.edu/JBI/docs/ReviewersManuals/Economic.pdf

Lima, M. D. D. P. D., Chaves, B. J. P., Lima, V. D. S., Silva, P. E., Soares, N. S. C. S., \& Santos, I. B. D. C. (2018). Riscos ocupacionais em profissionais de enfermagem de centros de material e esterilização. Revista Cuidarte, 9(3), 2361-2368. http://dx.doi.org/10.15649/cuidarte.v9i3.544

Lima Silva, M., Silva, M. P. B., Leite, A. C., de Oliveira, E. F. P., Fernandes, A. R. C. A., Jatobá, D. N. V., \& de Azevedo Freire, E. C. (2021). A ergonomia no ambiente de trabalho dos enfermeiros do samu: uma visão da enfermagem. Research, Society and Development, 10(1), e30410111552e30410111552. DOI: http://dx.doi.org/10.33448/rsd-v10il.11552

Mendes, K. D. S., Silveira, R. C. D. C. P., \& Galvão, C. M. (2008). Revisão integrativa: método de pesquisa para a incorporação de evidências na saúde e na enfermagem. Texto \& contexto-enfermagem, 17, 758-764. https://www.scielo.br/j/tce/a/XzFkq6tjWs4wHNqNjKJLkXQ/?lang=pt\&format=pdf

OIT, Organización Internacional del Trabajo. (2013). La prevención de las enfermidades profesionales. (2013). https://www.ilo.org/wcmsp5/groups/public/--ed_protect/---protrav/---safework/documents/publication/wcms_209555.pdf

Oliveira, L. B., Guimaraes, M. D. S. O., da Cruz Silva, W., Silva, G. B., de Sousa, A. F. L., \& Moura, M. E. B. (2014). Saúde e segurança do enfermeiro: riscos ergonômicos nos setores críticos do ambiente hospitalar. Revista de Enfermagem UFPE on line, 8(8), 2633-2637. 10.5205/reuol.6081-52328-1- SM.0808201408

Organização Mundial da Saúde (OMS). (2010). Ambientes de trabalho saudáveis: um modelo para ação: para empregadores, trabalhadores, formuladores de política e profissionais. https://www.who.int/occupational_health/ambientes_de_trabalho.pdf

Rogers, B., Buckheit, K., \& Ostendorf, J. (2013). Ergonomics and nursing in hospital environments. Workplace health \& safety, 61(10), 429-439. https://journals.sagepub.com/doi/pdf/10.1177/216507991306101003

Sanchez, M. L., Silveira, R. S. D., Figueiredo, P. P. D., Mancia, J. R., Schwonke, C. R. G. B., \& Gonçalves, N. G. D. C. (2018). Estratégias que contribuem para a visibilidade do trabalho do enfermeiro na central de material e esterilização. Texto \& Contexto-Enfermagem, 27. DOI: https://doi.org/10.1590/010407072018006530015

Santos, A. O., do Espírito Santo, I. M. B., da Silva, H. L. L., de Araújo Bezerra, A. M. F., dos Santos, J. F. C., de Holanda Lira, E. V., \& de Lima Fontes, F. L. (2021). Riscos ergonômicos aos quais a equipe de Enfermagem está exposta em suas práticas laborais. Research, Society and Development, 10(3), e24610313259-e24610313259. DOI: http://dx.doi.org/10.33448/rsd-v10i3.13259

Santos, M. R., Ribeiro, R. P., Martins, M. B., Do Nascimento, L. A., Martins, J. T., \& BoBroff, M. C. C. (2012). Avaliação da implantação da Norma Regulamentadora $32 \mathrm{em}$ um Hospital universitário. Cogitare Enfermagem, 17(3). https://revistas.ufpr.br/cogitare/article/view/25754/19045

SOBECC, Associação Brasileira de enfermeiros de centro cirúrgico, recuperação anestésica e centro de material e esterilização. (2017). Diretrizes de práticas em enfermagem cirúrgica e processamento de produtos para saúde. (7a ed.), https://sobecc.org.br/uploads/files/2020/12/diretrizes-1607560930.pdf

Sousa, F. M. S. D. (2011). Condições de trabalho de ambiente cirúrgico e a saúde dos trabalhadores de enfermagem. 116 f. Dissertação (Mestrado em Enfermagem). Universidade do Estado do Rio de Janeiro, https://www.bdtd.uerj.br:8443/bitstream/1/11212/1/ DISSERTACAO FINAL_ Fatima_Maria_ Saldanha_de_Sousa.pdf 